\title{
A Consistent Fourth-Order Compact Finite Difference Scheme for Solving Vorticity-Stream Function Form of Incompressible Navier-Stokes Equations
}

\author{
Tao Wang ${ }^{1,2}$ and Tiegang Liu ${ }^{1, *}$ \\ ${ }^{1}$ School of Mathematics and Systems Science, Beihang University, Beijing 100191, \\ China \\ 2 School of Mathematics and Information Science, North Minzu University, \\ Yinchuan 750021, China
}

Received 22 March 2018; Accepted (in revised version) 15 June 2018

\begin{abstract}
The inconsistent accuracy and truncation error in the treatment of boundary usually leads to performance defects, such as decreased accuracy and even numerical instability, of the entire computational method, especially for higher order methods. In this work, we construct a consistent fourth-order compact finite difference scheme for solving two-dimensional incompressible Navier-Stokes (N-S) equations. In the proposed method, the main truncation error term of the boundary scheme is kept the same as that of the interior compact finite difference scheme. With such a feature, the numerical stability and accuracy of the entire computation can be maintained the same as the interior compact finite difference scheme. Numerical examples show the effectiveness and accuracy of the present consistent compact high order scheme in $L^{\infty}$. Its application to two dimensional lid-driven cavity flow problem further exhibits that under the same condition, the computed solution with the present scheme is much close to the benchmark in comparison to those from the $4^{\text {th }}$ order explicit scheme. The compact finite difference method equipped with the present consistent boundary technique improves much the stability of the whole computation and shows its potential application to incompressible flow of high Reynolds number.
\end{abstract}

AMS subject classifications: 65Y04, 65Z05, 65N06, 65D25

Key words: Navier-Stokes equations, compact finite difference scheme, consistent boundary scheme, Lid-driven cavity.

\section{Introduction}

It is desirable to use higher order numerical methods to solve complex flow problems in many applications, such as those governed by incompressible Navier-Stokes (N$\mathrm{S})$ equations, due to their lower numerical diffusion and dispersion. Because of its compact

${ }^{*}$ Corresponding author. Email address: liutg@buaa.edu.cn (T. G. Liu) 
stencils among high order methods, there is consistent interest in developing compact finite difference method for solving convection-diffusion problems and N-S equations [1-7] since Adam, Kreiss and Hirsh [8] put forward the Hermitian three-point fourth-order compact scheme. One of the followed pioneering work was done by Lele [9], where a class of high order centered and linear compact schemes was proposed with high order boundary schemes suggested. A centered compact scheme is usually very effective for solving diffusion dominant problems with uniform mesh but encounters difficulty when applied to convection dominant problems.

With this regard, $\mathrm{Fu}$ and $\mathrm{Ma}$ [10] investigated the upwind compact finite difference approximation for solving convective dominant equations. Later, Tian [13] explored a kind of fourth-order explicit upwind compact difference schemes. In order to apply a compact scheme to nonuniform mesh, Chu and Fan $[11,12]$ tried to construct a sixth-order compact scheme via jointing the uniform and non-uniform grids. And Zhang et al. [14] developed a sixth-order compact scheme on staggered grids.

High-order compact finite difference schemes require additional numerical boundary schemes to treat the grid points near boundaries of the computational domain. Although it has proven that for a $p$ th-order scheme,the accuracy of boundary scheme can be one order lower, i.e. $(p-1)$ th-order, than that of the interior scheme in order to maintain the global accuracy of the entire computation [16] under $L^{2}$-norm, boundary treatment is still the major challenging of applying high order methods in engineering applications. The primary difficulty in using higher order compact finite difference schemes is to identify boundary schemes that are able to preserve the accuracy and the stability as well over the whole computational domain. For hyperbolic systems, Carpenter [17] introduced the fourth and sixth order compact schemes with Lele's boundary scheme [9] and a proposed sixth order boundary scheme, respectively. Recently, Liu et al. [15] also exhibited a class of centered compact finite difference schemes and the corresponding boundary schemes. For the two-dimensional vorticity-stream function form of incompressible N-S equations, E and Liu $[18,19]$ put forward a kind of fourth-order accuracy schemes, with both interior and boundary schemes to fourth-order accuracy.

We noticed that in all of those work mentioned above, the authors focused on developing some boundary schemes with the same order accuracy as that of the interior scheme, they did not pay much attention on numerical stability of the entire method. We have found recently that some of the popular boundary schemes can suffer numerical instability when applied to flow in high Reynolds number and the numerical stability of the entire computation is closely related to the coefficients of the leading truncation errors of both interior and boundary schemes.

This paper is primarily aimed at developing a kind of consistent fourth-order compact finite difference scheme to solve vorticity-stream function form of the two-dimensional incompressible Navier-Stokes equations. We present a fourth-order compact scheme for the boundary computation, in which the order of accuracy and leading truncation error term is designed to be the same as that of the interior scheme so that both the accuracy and stability can be kept during the whole computation. 


\section{Numerical method}

\subsection{Governing equations}

The two-dimensional incompressible Navier-Stokes equations are given in the vorticitystream function form as

$$
\begin{aligned}
& \frac{\partial^{2} \psi}{\partial x^{2}}+\frac{\partial^{2} \psi}{\partial y^{2}}=-\xi \\
& \frac{\partial \xi}{\partial t}+u \frac{\partial \xi}{\partial x}+v \frac{\partial \xi}{\partial y}=\frac{1}{R e}\left(\frac{\partial^{2} \xi}{\partial x^{2}}+\frac{\partial^{2} \xi}{\partial y^{2}}\right)+f
\end{aligned}
$$

where $\Omega$ is a rectangle and $(x, y) \in \Omega$. $\xi$ is the vorticity, $\psi$ is the stream function, $f$ is a prescribed forcing function, Re is the Reynolds number. The velocity components are defined as $u(x, y)=\frac{\partial \psi}{\partial y}$ and $v(x, y)=-\frac{\partial \psi}{\partial x}$. Eq. (2.1a) is referred to as the stream function equation and Eq. (2.1b) as the vorticity equation.

\subsection{Numerical scheme for the convection-diffusion equation}

We first develop the compact finite difference scheme for solving Eq. (2.1b), which is a $2 \mathrm{D}$ convection-diffusion equation and written as following

$$
\begin{aligned}
& \frac{\partial \xi}{\partial t}=L(\xi), \\
& L(\xi)=\frac{1}{R e}\left(\frac{\partial^{2} \xi}{\partial x^{2}}+\frac{\partial^{2} \xi}{\partial y^{2}}\right)-u \frac{\partial \xi}{\partial x}-w \frac{\partial \xi}{\partial y}+f .
\end{aligned}
$$

The spatial discretization is carried out on a uniform grid of width $h$ in both $x$ and $y$ directions. We use $\xi_{i j}$ to represent value of $\xi$ at grid node $\left(x_{i}, y_{j}\right), \xi_{x i j}$ and $\xi_{x x i j}$ to represent approximations of $\frac{\partial \xi}{\partial x}, \frac{\partial^{2} \xi}{\partial x^{2}}$ at node $\left(x_{i}, y_{j}\right)$, respectively. $\xi_{y i j}$ and $\xi_{y y i j}$ represents approximations of $\frac{\partial \xi}{\partial y}, \frac{\partial^{2} \xi}{\partial y^{2}}$ at node $\left(x_{i}, y_{j}\right)$, respectively, $i, j=1, \cdots, N+1$.

\subsubsection{Interior scheme}

In this work, Eq. (2.1b) will be solved with fourth order compact difference method. The second partial derivatives $\frac{\partial^{2} \xi}{\partial x^{2}}$ and $\frac{\partial^{2} \xi}{\partial y^{2}}$ in the diffusive term can be computed with the following fourth-order symmetrical compact schemes [8]:

$$
\begin{aligned}
& \frac{1}{12} \xi_{x x i-1 j}+\frac{5}{6} \xi_{x x i j}+\frac{1}{12} \xi_{x x i+1 j}=\frac{1}{h^{2}}\left(\xi_{i-1 j}-2 \xi_{i j}+\xi_{i+1 j}\right), \\
& \frac{1}{12} \xi_{y y i j-1}+\frac{5}{6} \xi_{y y i j}+\frac{1}{12} \xi_{y y i j+1}=\frac{1}{h^{2}}\left(\xi_{i j-1}-2 \xi_{i j}+\xi_{i j+1}\right) .
\end{aligned}
$$

It is noted that the truncation error of scheme $(2.3 \mathrm{a})$ is $-\frac{1}{240} h^{4} \xi_{i j}^{(6)}+\mathscr{O}\left(h^{6}\right)$. 
The first partial derivatives of $\frac{\partial \xi}{\partial x}$ and $\frac{\partial \xi}{\partial y}$ in the convective terms can be computed with fourth-order symmetrical compact schemes [8].

$$
\begin{aligned}
& \frac{1}{6} \xi_{x i-1 j}+\frac{2}{3} \xi_{x i j}+\frac{1}{6} \xi_{x i+1 j}=\frac{1}{2 h}\left(\xi_{i-1 j}-\xi_{i+1 j}\right), \\
& \frac{1}{6} \xi_{y i j-1}+\frac{2}{3} \xi_{y i j}+\frac{1}{6} \xi_{y i j+1}=\frac{1}{2 h}\left(\xi_{i j-1}-\xi_{i j+1}\right) .
\end{aligned}
$$

The velocities, $u$ and $v$, can be viewed as the first partial derivatives of $\psi$, thus they can also be computed with Eq. (2.4a) and Eq. (2.4b). However, the use of Eq. (2.4a) and Eq. (2.4b) to discrete the nonlinear convective terms of $u \frac{\partial \xi}{\partial x}$ and $v \frac{\partial \xi}{\partial y}$ in Eq. (2.1b) would reveal poor performance to strong convective flow. Therefore, we employ the upwind compact scheme to discretize the nonlinear convective terms, $u \frac{\partial \xi}{\partial x}$ and $v \frac{\partial \xi}{\partial y}$.

$$
u \frac{\partial \xi}{\partial x}=u^{+} \xi_{x i j}^{+}+u^{-} \xi_{x i j}^{-}, \quad u^{+}=\frac{u+|u|}{2}, \quad u^{-}=\frac{u-|u|}{2} .
$$

The fourth-order upwind compact scheme proposed in [13] for $\xi_{x i j}^{+}$and $\xi_{x i j}^{-}$is employed and expressed as

$$
\begin{aligned}
& \xi_{x i-1 j}^{+}+2 \xi_{x i j}^{+}=\frac{1}{2 h}\left(-7 \xi_{i-1 j}+8 \xi_{i j}-\xi_{i+1 j}\right)+h \xi_{x x i j}, \\
& 2 \xi_{x i j}^{-}+\xi_{x i+1 j}^{-}=\frac{1}{2 h}\left(\xi_{i-1 j}-8 \xi_{i j}+\xi_{i+1 j}\right)-h \xi_{x x i j} .
\end{aligned}
$$

We notice that the truncation error of scheme (2.6a) is $-\frac{1}{60} h^{4} \xi_{i j}^{(5)}-\frac{1}{360} h^{5} \xi_{i j}^{(6)}+\mathscr{O}\left(h^{6}\right)$.

\subsubsection{Boundary scheme}

To solve the convection-diffusion equation with the above interior scheme, computation of the first and second derivatives of $\xi$ at boundary points, $i=1, N+1$, have to be formulated in advance, leading to the construction of boundary scheme.

In literature, many authors focused on proposing their high precision interior schemes, while adopting a low accuracy boundary schemes according to the theory in [16]. In [9], Lele introduced the second-, third- and fourth-order implicit boundary schemes for the first and second order derivatives. Especially when fourth-order accuracy interior points schemes are used, the third order explicit difference scheme is popularly employed for the boundary points. In fact, for many boundary value problems, it is found that lower order difference schemes for boundaries can affect the entire accuracy no matter how higher order difference scheme are used for the interior points. Developing high order difference schemes for the boundary points which consistently matches the interior points schemes is the target of this subsection.

Definition 2.1 (Definition 1). A boundary scheme is called to be consistent with the interior scheme, if 


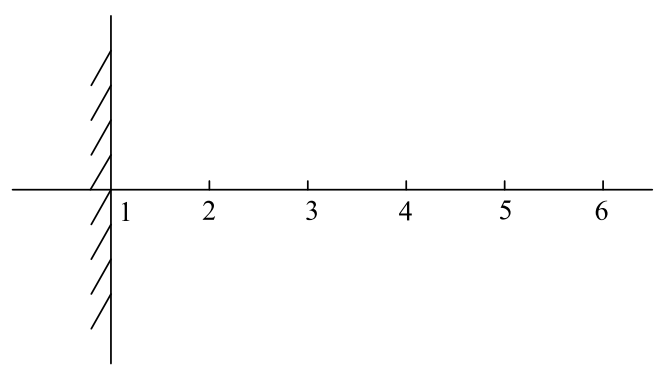

Figure 1: Computational stencil for left boundary nodes.

(i) the order of accuracy of the boundary scheme is same as the interior scheme;

(ii) the major truncation error term of the boundary scheme is identical to that of the interior scheme;

(iii) the coefficient matrix of algebraic system is strictly diagonally dominant, which guaranteed the stability of the coupling computational system of interior and boundary schemes.

We call such a scheme is a consistent boundary scheme.

Definition 2.2 (Definition 2). We say the entire method is consistent if a consistent boundary scheme is applied together with the stable interior scheme.

From the above definition, it is easy to conclude that the entire computation is numerically stable if a consistent scheme is applied. Below, we take the fourth-order compact finite difference scheme as an example to show how to construct a consistent boundary scheme for the compact finite difference scheme.

We first give the second derivative of the consistent fourth-order boundary schemes. Without loss of generality, we derived the fourth order accuracy boundary schemes by using Taylor series expansion with undetermined coefficients.

We express the left boundary schemes as

$$
\xi_{1}^{\prime \prime}+\beta_{1} \xi_{2}^{\prime \prime}=\frac{1}{h^{2}}\left(b_{1} \xi_{1}+b_{2} \xi_{2}+b_{3} \xi \xi_{3}+b_{4} \xi_{4}+b_{5} \xi_{5}+b_{6} \xi_{6}\right),
$$

where the notation in Fig. 1 is used. We require scheme (2.7) to be fourth order accurate, we obtain

$$
\begin{array}{lll}
b_{1}=\frac{5}{6} \beta_{1}+\frac{15}{4}, & b_{2}=-\frac{5}{4} \beta_{1}-\frac{77}{6}, & b_{3}=-\frac{1}{3} \beta_{1}+\frac{107}{6} \\
b_{4}=\frac{7}{6} \beta_{1}-13, & b_{5}=-\frac{1}{2} \beta_{1}+\frac{61}{12}, & b_{6}=\frac{1}{12} \beta_{1}-\frac{10}{12} .
\end{array}
$$

The truncation error of the boundary scheme (2.7) is $\left(\frac{13}{180} \beta_{1}-\frac{137}{180}\right) h^{4} \xi_{1}^{(6)}+\mathscr{O}\left(h^{5}\right)$.

Scheme (2.7) is the usually form applied to a fourth order interior scheme to treat the boundary. It includes two famous boundary schemes. When $\beta_{1}=10$ and $b_{6}=0$, the 
boundary scheme is the $4^{\text {th }}$ order implicit scheme proposed by Lele [9] and expressed as

$$
\xi_{1}^{\prime \prime}+10 \xi_{2}^{\prime \prime}=\frac{1}{h^{2}}\left(\frac{145}{12} \xi_{1}-\frac{76}{3} \xi_{2}+\frac{29}{2} \xi_{3}-\frac{4}{3} \xi_{4}+\frac{1}{12} \xi_{5}\right) \text {. }
$$

The truncation error of scheme (2.8) is $-\frac{7}{180} h^{4} \xi_{1}^{(6)}+\mathscr{O}\left(h^{5}\right)$, which is inconsistent with that of the above interior scheme. The boundary scheme (2.8) is actually unstable when coupled with the above interior scheme as we will show in the section of numerical examples, because the system coefficient matrix of the jointed boundary and interior schemes is not diagonally dominant.

In scheme (2.7), when $\beta_{1}=0$, the boundary scheme is a $4^{\text {th }}$ order explicit scheme [9] as

$$
\xi_{1}^{\prime \prime}=\frac{1}{h^{2}}\left(\frac{45}{4} \xi_{1}-\frac{77}{6} \xi_{2}+\frac{107}{6} \xi_{3}-13 \xi_{4}+\frac{61}{12} \xi_{5}-\frac{10}{12} \xi_{6}\right) .
$$

The truncation error of scheme (2.9) is $-\frac{137}{180} h^{4} \xi_{1}^{(6)}+\mathscr{O}\left(h^{5}\right)$, which is not identical to that of the above interior scheme. Numerical oscillations can occur to scheme (2.9) if the Reynolds number is high as to be shown in the section of numerical results.

If we require the system is stable when the left boundary scheme (2.7) coupled with the above interior scheme, i.e. the coefficient matrix of the resultant system is diagonally dominant, the $\beta$ appearing in scheme (2.7) must satisfy $-1<\beta_{1}<1$. If scheme (2.7) is strictly limited to be consistent with the above interior scheme, it requires that

$$
\frac{13}{180} \beta_{1}-\frac{137}{180}=-\frac{1}{240} \Rightarrow \beta_{1}=\frac{545}{52},
$$

then $\beta_{1}>1$. Consequently, we have following conclusion.

Theorem 2.1. The popular boundary scheme (2.7) is inconsistent when applied with the interior scheme of fourth order compact finite difference scheme.

To obtain a consistent boundary scheme, we add one more free parameter to the boundary scheme as

$$
\xi_{1}^{\prime \prime}+\beta_{1} \xi_{2}^{\prime \prime}=\frac{1}{h^{2}}\left(b_{1} \xi_{1}+b_{2} \xi_{2}+b_{3} \xi_{3}+b_{4} \xi_{4}+b_{5} \xi_{5}+b_{6} \xi_{6}+b_{7} \xi_{7}\right) .
$$

We require the scheme to be fourth order accurate, we have

$$
\begin{aligned}
& b_{1}=\frac{5}{6} \beta_{1}+\frac{15}{4}+b_{7}, \quad b_{2}=-\frac{5}{4} \beta_{1}-\frac{77}{6}-6 b_{7}, \quad b_{3}=-\frac{1}{3} \beta_{1}+\frac{107}{6}+15 b_{7}, \\
& b_{4}=\frac{7}{6} \beta_{1}-13-20 b_{7}, \quad b_{5}=-\frac{1}{2} \beta_{1}+\frac{61}{12}+15 b_{7}, \quad b_{6}=\frac{1}{12} \beta_{1}-\frac{10}{12}-6 b_{7} \text {. }
\end{aligned}
$$

The truncation error of scheme (2.10) is

$$
\left(\frac{13}{180} \beta_{1}-\frac{137}{180}+b_{7}\right)(h)^{4} \xi_{1}^{(6)}+\left(\frac{7}{45} \beta_{1}-\frac{19}{12}+3 b_{7}\right)(h)^{5} \xi_{1}^{(7)}+\mathscr{O}\left(h^{6}\right)
$$


in which there are two parameters $\beta_{1}, b_{7}$ to choose freely.

To meet the condition (ii), (iii) in definition 1 , we have

$$
\left\{\begin{array}{l}
\frac{13}{180} \beta_{1}-\frac{137}{180}+b_{7}=-\frac{1}{240} \\
-1<\beta_{1}<1
\end{array}\right.
$$

which results in $\frac{493}{720}<b_{7}<\frac{597}{720}$. Thus, there are consistent boundary schemes with expression of (2.10)

Among $\frac{493}{720}<b_{7}<\frac{597}{720}$, we require the second major truncation error term becomes much smaller, one of the choice is $b_{7}=\frac{494}{720}$ and $\beta_{1}=\frac{51}{52}$.

At this point, the left boundary fourth-order scheme of the second derivative becomes

$$
\begin{aligned}
\xi_{1}^{\prime \prime}+\frac{51}{52} \xi_{2}^{\prime \prime}=\frac{1}{h^{2}} & \left(\frac{12293}{2340} \xi_{1}-\frac{18903}{1040} \xi_{2}+\frac{2891}{104} \xi_{3}-\frac{23941}{936} \xi_{4}\right. \\
+ & \left.\frac{387}{26} \xi_{5}-\frac{5063}{1040} \xi_{6}+\frac{247}{360} \xi_{7}\right) .
\end{aligned}
$$

The truncation error of the scheme (2.11) becomes $-\frac{1}{240} h^{4} \xi_{1}^{(6)}+\frac{979}{1560} h^{5} \xi_{1}^{(7)}+\mathscr{O}\left(h^{6}\right)$.

Similarly, we can develop the right boundary scheme of fourth order accuracy as

$$
\begin{aligned}
\xi_{N+1}^{\prime \prime}+\beta_{2} \xi_{N}^{\prime \prime}=\frac{1}{h^{2}} & \left(b_{N+1} \xi_{N+1}+b_{N} \xi_{N}+b_{N-1} \xi_{N-1}+b_{N-2} \xi_{N-2}\right. \\
& \left.+b_{N-3} \xi_{N-3}+b_{N-4} \xi_{N-4}+b_{N-5} \xi_{N-5}\right)
\end{aligned}
$$

with

$$
\begin{array}{ll}
b_{N+1}=\frac{5}{6} \beta_{2}+\frac{15}{4}+b_{N-5}, & b_{N}=-\frac{5}{4} \beta_{2}-\frac{77}{6}-6 b_{N-5}, \\
b_{N-1}=-\frac{1}{3} \beta_{2}+\frac{107}{6}+15 b_{N-5}, & b_{N-2}=\frac{7}{6} \beta_{2}-13-20 b_{N-5}, \\
b_{N-3}=-\frac{1}{2} \beta_{2}+\frac{61}{12}+15 b_{N-5}, & b_{N-4}=\frac{1}{12} \beta_{2}-\frac{10}{12}-6 b_{N-5}, \\
b_{N-5}=b_{N-5} . &
\end{array}
$$

Taking $\beta_{2}=-\frac{51}{52}$ and $b_{N-5}=\frac{596}{720}$, we have the fourth-order right boundary scheme of the second derivative as

$$
\begin{aligned}
\xi_{N+1}^{\prime \prime}-\frac{51}{52} \xi_{N}^{\prime \prime}=\frac{1}{h^{2}} & \left(\frac{17599}{4680} \xi_{N+1}-\frac{17237}{1040} \xi_{N}+\frac{795}{26} \xi_{N-1}-\frac{28735}{936} \xi_{N-2}\right. \\
& \left.+\frac{1871}{104} \xi_{N-3}-\frac{6117}{1040} \xi_{N-4}+\frac{149}{180} \xi_{N-5}\right) .
\end{aligned}
$$

The truncation error of scheme (2.13) is $-\frac{1}{240} h^{4} \xi_{N+1}^{(6)}-\frac{583}{780} h^{5} \xi_{N+1}^{(7)}+\mathscr{O}\left(h^{6}\right)$.

In summary, we can get the following theorem. 
Theorem 2.2. (1) There are consistent fourth-order boundary schemes for the second derivative; (2) In order to ensure that the main truncation error of the scheme is consistent, the scheme must with at least two degrees of freedom.

Next, we derive the consistent fourth-order boundary schemes for treating the first derivative of $\xi$. Similarly, we can prove that the popular form of

$$
\xi_{1}^{\prime}+\alpha \xi_{2}^{\prime}=\frac{1}{h}\left(a_{1} \xi_{1}+a_{2} \xi_{2}+a_{3} \xi_{3}+a_{4} \xi_{4}+a_{5} \xi_{5}\right)
$$

does not permit consistent boundary scheme. The boundary scheme (2.14) includes the popular $4^{\text {th }}$ order implicit scheme $\left(\alpha=3\right.$ and $\left.a_{5}=0\right)$ and the $4^{\text {th }}$ order explicit $(\alpha=0)$ proposed in [9].

To obtain a consistent scheme, the left boundary schemes can be written as

$$
\xi_{1}^{\prime}+\alpha \xi_{2}^{\prime}=\frac{1}{h}\left(a_{1} \xi_{1}+a_{2} \xi_{2}+a_{3} \xi_{3}+a_{4} \xi_{4}+a_{5} \xi_{5}+a_{6} \xi_{6}\right)
$$

with

$$
\begin{array}{lll}
a_{1}=-\frac{1}{4} \alpha_{1}-\frac{25}{12}-a_{6}, & a_{2}=-\frac{5}{6} \alpha_{1}+4+5 a_{6}, & a_{3}=\frac{3}{2} \alpha_{1}-3-10 a_{6}, \\
a_{4}=-\frac{1}{2} \alpha_{1}+\frac{4}{3}+10 a_{6}, & a_{5}=\frac{1}{12} \alpha_{1}-\frac{1}{4}-5 a_{6}, & a_{6}=a_{6}
\end{array}
$$

for the fourth order accuracy. Taking $\alpha=\frac{14}{15}$ and $a_{6}=\frac{41}{300}$, we have the left boundary fourth-order scheme for the first derivative as

$$
\xi_{1}^{\prime}+\frac{14}{15} \xi_{2}^{\prime}=\frac{1}{h}\left(-\frac{184}{75} \xi_{1}+\frac{703}{180} \xi_{2}-\frac{89}{30} \xi_{3}+\frac{67}{30} \xi_{4}-\frac{77}{90} \xi_{5}+\frac{41}{300} \xi_{6}\right)
$$

The corresponding truncation error of scheme (2.16) is $-\frac{1}{60} h^{4} \xi_{1}^{(5)}+\frac{169}{1800} h^{5} \xi_{1}^{(6)}+\mathscr{O}\left(h^{6}\right)$.

The right boundary scheme is

$$
\begin{aligned}
\xi_{N+1}^{\prime}+\alpha_{2} \xi_{N}^{\prime}=\frac{1}{h} & \left(a_{N+1} \xi_{N+1}+a_{N} \xi_{N}+a_{N-1} \xi_{N-1}+a_{N-2} \xi_{N-2}\right. \\
& \left.+a_{N-3} \xi_{N-3}+a_{N-4} \xi_{N-4}\right) .
\end{aligned}
$$

With $\alpha_{2}=-\frac{14}{15}$ and $a_{N-4}=-\frac{23}{100}$, the fourth-order right boundary scheme of the first derivative can be expressed as

$$
\begin{aligned}
& \xi_{N+1}^{\prime}-\frac{14}{15} \xi_{N}^{\prime} \\
= & \frac{1}{h}\left(\frac{52}{25} \xi_{N+1}-\frac{1067}{180} \xi_{N}+\frac{67}{10} \xi_{N-1}-\frac{41}{10} \xi_{N-2}+\frac{133}{90} \xi_{N-3}-\frac{23}{100} \xi_{N-4}\right) .
\end{aligned}
$$

The truncation error of scheme (2.18) is then $-\frac{1}{60} h^{4} \xi_{N+1}^{(5)}-\frac{281}{1800} h^{5} \xi_{N+1}^{(6)}+\mathscr{O}\left(h^{6}\right)$.

In summary, we have the following theorem. 
Theorem 2.3. (1) There are consistent fourth-order boundary schemes for the first derivative; (2)In order to ensure that the main truncation error is consistent, the scheme must have at least two degrees of freedom.

After spatial discretization with above method, the semi-discrete scheme is equivalent to the first ODE system. For the discretization in time, the explicit third order TVD RungeKutta scheme (for the details, please see [20]) was used. It is given as

$$
\begin{aligned}
& \xi^{(1)}=\alpha_{1} \xi^{n}+\beta_{1} \tau L\left(\xi^{n}\right), \\
& \xi^{(2)}=\alpha_{2} \xi^{n}+\beta_{2}\left[\xi^{(1)}+\tau L\left(\xi^{(1)}\right)\right], \\
& \xi^{n+1}=\alpha_{3} \xi^{n}+\beta_{3}\left[\xi^{(2)}+\tau L\left(\xi^{(2)}\right)\right],
\end{aligned}
$$

where $\tau$ stands for the time stepsize, $\alpha_{1}=1, \alpha_{2}=\frac{3}{4}, \alpha_{3}=\frac{1}{3}, \beta_{1}=1, \beta_{2}=\frac{1}{4}$ and $\beta_{3}=\frac{2}{3}$.

\subsection{Numerical scheme for Poisson-type equation}

Eq. (2.1a) for the stream function $\psi$ is a Poisson-type equation. Following [21], a fourth-order symmetric scheme for Eq. (2.1a) is applied

$$
\begin{aligned}
& \frac{2}{h^{2}}\left[\left(\psi_{i+1 j+1}+\psi_{i+1 j-1}+\psi_{i-1 j+1}+\psi_{i-1 j-1}-20 \psi_{i j}\right)\right. \\
& \left.\quad+4\left(\psi_{i+1 j}+\psi_{i-1 j}+\psi_{i j+1}+\psi_{i j-1}\right)\right] \\
& =-\left(8 \xi_{i j}+\xi_{i+1 j}+\xi_{i-1 j}+\xi_{i j+1}+\xi_{i j-1}\right) .
\end{aligned}
$$

Eq. (2.20) may be solved by using various efficient iterative techniques. In this paper, a line iterative approach with an alternating direction implicit (ADI) procedure enables us to obtain the solutions of Eq. (2.20) by applying of the tridiagonal matrix algorithm (TDMA) in each direction. This leads to a considerable saving in computing time.

\section{Numerical experiments}

In this section, we perform numerical experiments to illustrate the accuracy and effectiveness of the proposed higher order consistent compact difference schemes. All results were run on a Lenovo computer using double precision arithmetic. In the discussion of numerical results in this section, "fourth (or 4th) order implicit scheme" and "fourth (or 4th) order explicit scheme" means the boundary scheme employed. The interior scheme is kept the same for all computation.

\subsection{Example 1}

We consider the following unsteady convection-diffusion of a two-dimensional scalar equation:

$$
\frac{\partial u}{\partial t}+0.1\left(\frac{\partial u}{\partial x}+\frac{\partial u}{\partial y}\right)=0.01\left(\frac{\partial^{2} u}{\partial x^{2}}+\frac{\partial^{2} u}{\partial y^{2}}\right), \quad(x, y) \in[0,1] \times[0,1]
$$


Table 1: The $L^{\infty}$ errors and the rate of the convergence at $t=1$. (Note:6.792( $(-3)=6.792 \times 10^{-3}$,etc.)

\begin{tabular}{||c|cc|cc|cc||}
\hline & \multicolumn{2}{|c|}{$4^{\text {th }}$ order implicit } & \multicolumn{2}{c||}{$4^{\text {th }}$ order explicit } & \multicolumn{2}{c||}{ present } \\
\hline Grid & $L^{\infty}$-error & Rate & $L^{\infty}$-error & Rate & $L^{\infty}$-error & Rate \\
\hline $11 \times 11$ & $6.792(-3)$ & - & $1.386(-2)$ & - & $4.856(-3)$ & - \\
$21 \times 21$ & $5.278(-4)$ & 3.685 & $4.310(-4)$ & 5.007 & $2.448(-4)$ & 4.311 \\
$31 \times 31$ & $2.684(-4)$ & 1.667 & $6.205(-5)$ & 4.780 & $4.441(-5)$ & 4.209 \\
$41 \times 41$ & $1.168(-4)$ & 2.892 & $1.668(-5)$ & 4.566 & $1.347(-5)$ & 4.146 \\
$51 \times 51$ & $6.823(-5)$ & 2.409 & $6.234(-6)$ & 4.410 & $5.385(-6)$ & 4.108 \\
\hline
\end{tabular}

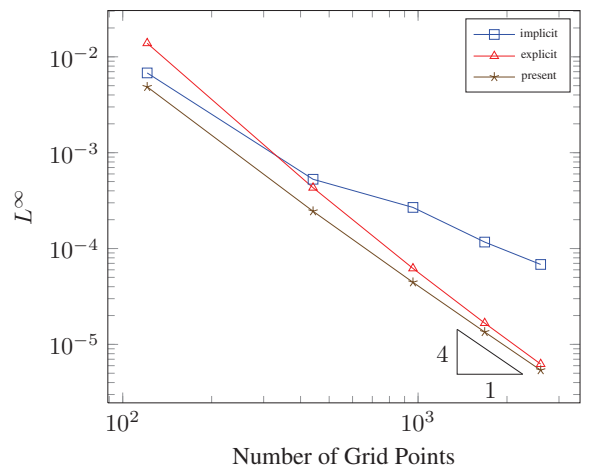

Figure 2: The $L^{\infty}$ - error convergence history of the three schemes.

The analytical solution to this equation is given as

$$
u(x, y, t)=e^{5(x+y)-t\left(0.02 \pi^{2}+0.5\right)} \cdot \sin (\pi(x+y)) .
$$

The initial and boundary conditions are directly obtained from its analytical solution.

Table 1 gives the $L^{\infty}$ error and the convergence rate for Example 1 by using the present consistent boundary scheme and other two inconsistent boundary schemes, i.e., $4^{\text {th }}$ order implicit scheme and $4^{\text {th }}$ order explicit scheme. It is observed that the consistent boundary scheme produces more accurate solution than $4^{\text {th }}$ order implicit scheme and $4^{\text {th }}$ order explicit scheme. It shows that the present scheme reached fourth-order accuracy in space.

Fig. 2 shows the convergent order of accuracy with mesh refinement. The fourth-order implicit scheme can not reach its optimal accuracy because of numerical instability.

\subsection{Example 2}

We consider the following linear Burgers equation

$$
\frac{\partial u}{\partial t}+\frac{\partial u}{\partial x}=v \frac{\partial^{2} u}{\partial x^{2}}, \quad x \in[0,1] .
$$

The initial condition is $u(x, 0)=0$, and the boundary conditions are $u(0, t)=1, u(1, t)=0$. The exact solution of this problem gives as $u(x)=\frac{1-e^{-\operatorname{Re}(1-x)}}{1-e^{-\operatorname{Re}}}$, where $\operatorname{Re}=\frac{1}{v}$. The solution 


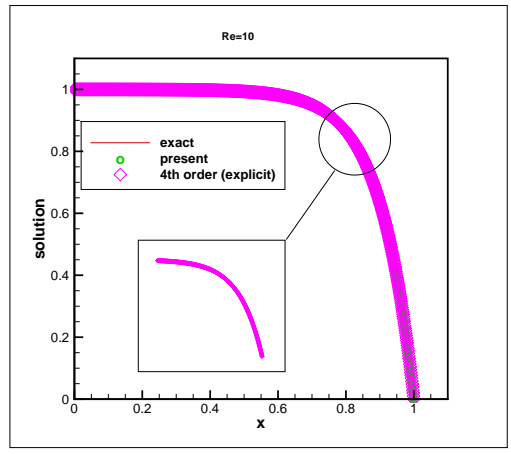

(a)

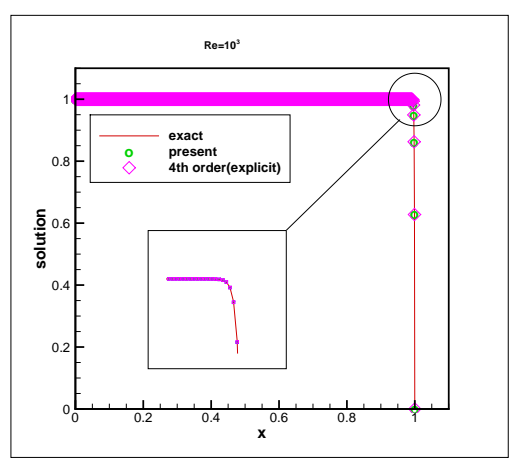

(c)

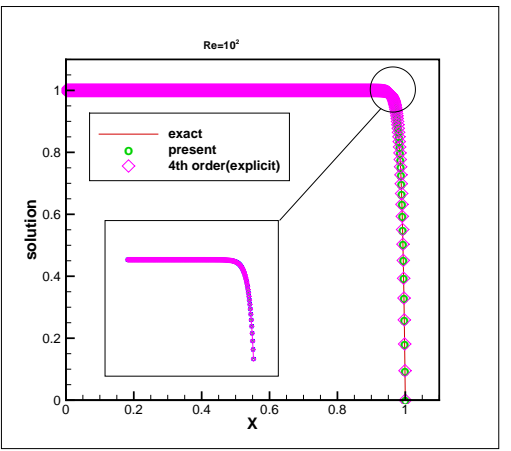

(b)

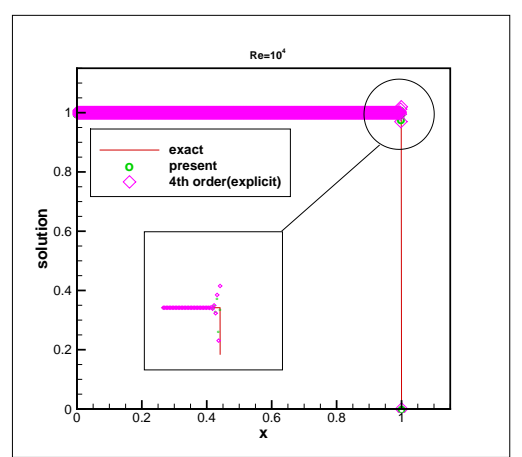

(d)

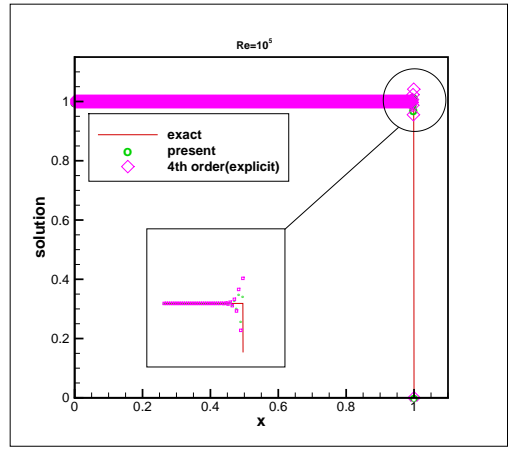

(e)

Figure 3: The exact and numerical solution with two boundary schemes with different Reynolds numbers: (a) $R e=10$; (b) $R e=10^{2}$; (c) $R e=10^{3}$; (d) $R e=10^{4}$; (e) $\operatorname{Re}=10^{5}$.

is regarded as steady when $\delta=\operatorname{Max}\left|u_{i}^{m+1}-u_{i}^{m}\right| \leq 10^{-12}$, where $m$ denote the time marching step.

A series of plots in Fig. 3 shows the numerical results obtained by using the present and $4^{\text {th }}$ order explicit schemes. We observe that when $10^{1} \leq R e \leq 10^{3}$, the two schemes produce perfect results because the smoothness of the exact solution is good. When $R e \geq$ $10^{4}$,numerical oscillation occurs to the fourth-order explicit scheme near boundary at 
Table 2: The errors with two boundary schemes with different Reynolds numbers.

\begin{tabular}{||c|cc|cc||}
\hline & \multicolumn{2}{|c|}{$4^{\text {th }}$ order explicit } & \multicolumn{2}{c||}{ present } \\
\hline $\operatorname{Re}$ & $L^{2}$-error & $L^{\infty}$-error & $L^{2}$-error & $L^{\infty}$-error \\
\hline $10^{1}$ & $1.565(-7)$ & $2.868(-7)$ & $1.542(-7)$ & $2.806(-7)$ \\
$10^{2}$ & $2.035(-8)$ & $1.245(-7)$ & $1.750(-8)$ & $1.038(-7)$ \\
$10^{3}$ & $1.555(-4)$ & $4.936(-3)$ & $1.173(-4)$ & $3.208(-3)$ \\
$10^{4}$ & $1.218(-3)$ & $3.023(-2)$ & $7.557(-4)$ & $2.202(-2)$ \\
$10^{5}$ & $2.115(-3)$ & $4.463(-2)$ & $1.173(-3)$ & $3.004(-2)$ \\
\hline
\end{tabular}

Table 3: The $L^{\infty}$ errors and the rate of the convergence for stream function at $R e=$ 100.(Note:div.=divergence.)

\begin{tabular}{||c|cc|cc|cc||}
\hline & \multicolumn{2}{|c|}{$4^{\text {th }}$ order implicit } & \multicolumn{2}{|c||}{$4^{\text {th }}$ order explicit } & \multicolumn{2}{c||}{ present } \\
\hline Grid & $\psi$-error & Rate & $\psi$-error & Rate & $\psi$-error & Rate \\
\hline $11 \times 11$ & div. & - & $2.402(-5)$ & - & $2.368(-5)$ & - \\
$21 \times 21$ & div. & - & $1.663(-6)$ & 3.852 & $1.644(-6)$ & 3.848 \\
$31 \times 31$ & div. & - & $3.379(-7)$ & 3.930 & $3.377(-7)$ & 3.904 \\
$41 \times 41$ & div. & - & $1.089(-7)$ & 3.934 & $1.089(-7)$ & 3.932 \\
\hline
\end{tabular}

$x=1$ as shown in Fig. 3(d) and (e). The present consistent fourth-order boundary scheme still gives good results. This validates that to keep the coefficient matrix of the whole scheme to be strictly diagonally dominant is an important factor to ensure the numerical stability. The performance of the present method is far superior to the fourth-order explicit scheme in both computational accuracy and resolution.

As further shown in Table 2 of the $L^{2}$ and $L^{\infty}$ errors with two boundary schemes with different Reynolds numbers, the consistent fourth-order boundary scheme is also better than the fourth-order explicit scheme.

\subsection{Example 3}

This is a steady analytical solution of Eq. (2.1b) constructed by Richards et al. [22] to assess the effects of grid staggering under $f=0$.

$$
\begin{aligned}
& \psi(x, y)=\frac{2}{R e} \cdot \tan ^{-1} \cdot \frac{y}{x}-\frac{1}{16}\left(x^{2}+y^{2}\right)^{2}, \\
& \xi(x, y)=x^{2}+y^{2} .
\end{aligned}
$$

The computational domain is $(x, y) \in[1,2] \times[1,2]$. The boundary conditions are given by the analytical values at boundary nodes.

Table 3 and Table 4 depict the $L^{\infty}$ error and the convergence rate for Example 3, by using the present scheme and the other two fourth-order accuracy boundary schemes with different mesh sizes. It shows that the present scheme and the fourth-order explicit scheme reached fourth order accuracy in space. The consistent boundary scheme produces more accurate solution than the two inconsistent boundary schemes. The fourth-order implicit 
Table 4: The $L^{\infty}$ errors and the rate of convergence for vorticity at $R e=100$.

\begin{tabular}{|c|c|c|c|c|c|c|}
\hline & \multicolumn{2}{|c|}{$4^{\text {th }}$ order implicit } & \multicolumn{2}{|c|}{$4^{\text {th }}$ order explicit } & \multicolumn{2}{|c|}{ present } \\
\hline Grid & $\xi$-error & Rate & $\xi$-error & Rate & $\xi$-error & Rate \\
\hline $11 \times 11$ & div. & - & $1.398(-4)$ & - & $1.281(-4)$ & \\
\hline $21 \times 21$ & div. & - & $8.546(-6)$ & 4.031 & $7.976(-6)$ & 4.005 \\
\hline $31 \times 31$ & div. & - & $1.471(-6)$ & 4.338 & $1.471(-6)$ & 4.168 \\
\hline $41 \times 41$ & div. & - & $4.077(-7)$ & 4.461 & $4.077(-7)$ & 4.461 \\
\hline
\end{tabular}

scheme cannot get convergence solution because its corresponding matrix of the jointed boundary scheme and interior scheme is not diagonally dominant.

\subsection{Application: Lid-Driven Cavity Flow}

We consider the problem of two-dimensional lid-driven cavity flow, which is extensively used to demonstrate the accuracy and efficiency of numerical methods for incompressible flows. This problem is of scientific interest because it displays almost all fluid mechanical phenomena for incompressible viscous flows in the simplest of geometric settings.

The computational domain is $(x, y) \in[0,1]$. The fluid motion is generated by the sliding motion of the top wall of the cavity at $y=1 \mathrm{from}$ left to right. Boundary conditions on the top wall are given as $u=1, v=0$. On all other walls of the cavity the velocities are zero $(u=v=0)$.

Further, the stream function values on all four walls are zero $(\psi=0)$. Due to the lack of the physical boundary conditions for the vorticity $\omega$ on all walls, numerical boundary of vorticity needs to be presented. Following [4], we have

$$
h\left(6 \xi_{1}+4 \xi_{2}-\xi_{3}\right) / 21+o\left(h^{4}\right)=\left(15 \psi_{1}-16 \psi_{2}+\psi_{3}\right) /(14 h) \pm V_{w},
$$

where $1,2,3$ is neighboring points and boundary adjacent points respectively, $V_{m}$ is for the wall of tangential velocity, on the sliding walls $V_{m}=1$, on the solid walls $V_{m}=0$.

Let

$$
\triangle U_{\max }(n \triangle t)=\max \left[\left(u_{i, j}^{n+1}-u_{i, j}^{n}\right)^{2}+\left(v_{i, j}^{n+1}-v_{i, j}^{n}\right)^{2}\right]^{1 / 2}<\varepsilon
$$

denote the root square error at the $(n+1)$ time level. When $\Delta U_{\max }(n \Delta t) \leq 10^{-8}$, the solution is qualified as steady.

Numerical solutions for the driven-cavity flow are obtained with different Reynolds numbers. In present computation, the grid mesh spacing is equal to $\frac{1}{64}$ and $\frac{1}{128}$ and the time increment is equal to 0.001 .

Fig. 4 exhibits the streamline contours for the cavity flows with the Reynolds numbers $1000,3200,5000,7500$. In these figures, the typical separation and secondary vortices at the bottom corners of the cavity as well as the top left can be seen. These stream function profiles are in very good agreement with the benchmark results of Ghia et al. [24] and other established results [25-28], which confirms the present method yields quantitatively accurate solutions. Fig. 5 show the vorticity contours with Reynolds numbers 


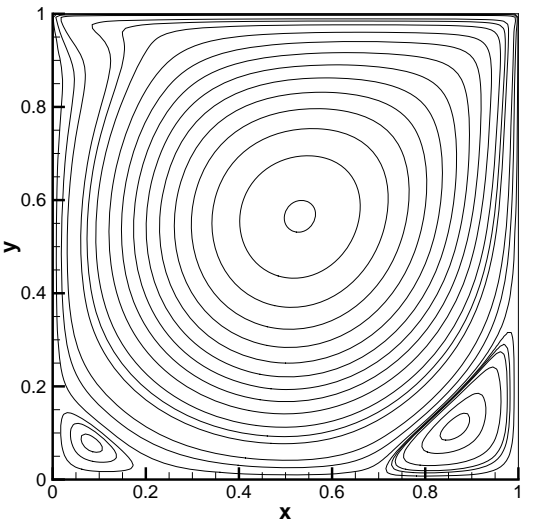

(a)

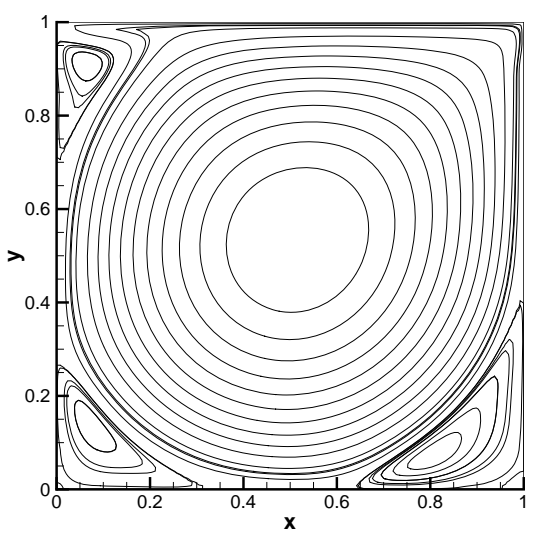

(c)

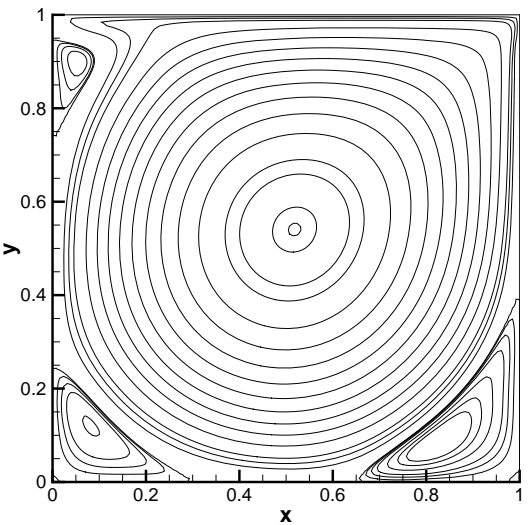

(b)

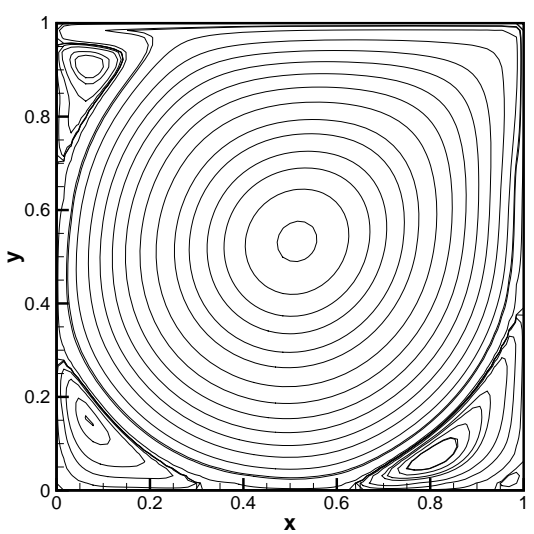

(d)

Figure 4: The steady-state streamline contours: (a) $R e=1000$; (b) $R e=3200$; (c) $R e=5000$; and (d) $\operatorname{Re}=7500$.

$1000,3200,5000,7500$, the vorticity contours provided with the our method agree well with those established results in [26]. Again,a pretty consistent comparison is obtained.

To validate the present method quantitatively,the minimum values $u_{\min }$ and the corresponding location coordinate $y_{\min }$ of horizontal velocity along the vertical centerline, the maximum values $v_{\max }$ and the corresponding location coordinate $x_{\max }$ of vertical velocity along the horizontal centerline, and the values of stream-function at the primary vortex center and the corresponding locations obtained numerically are collected and listed in Table 5 for different Reynolds numbers $(1000 \leq R e \leq 7500)$. The available comparison data from the literature are also given in this table. In Table 6, the location of the center of secondary vortices and the value of stream-function at the vortex center obtained in the present computation are compared with data from the literature $[24,25,27]$ with the Reynolds numbers of 1000, 3200, 5000 and 7500, respectively. It is clear from all these comparisons that the results of the present numerical method are reliable . 


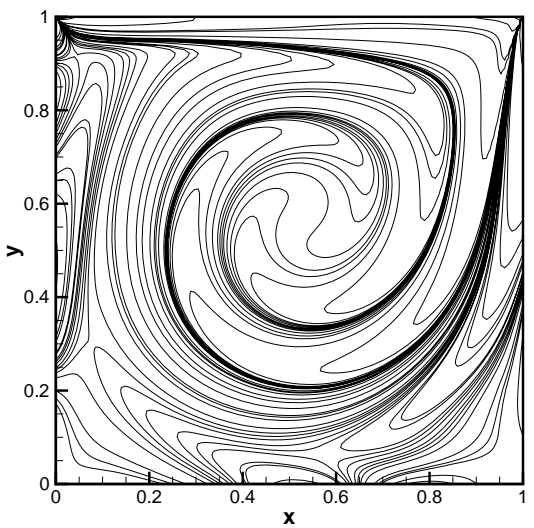

(a)

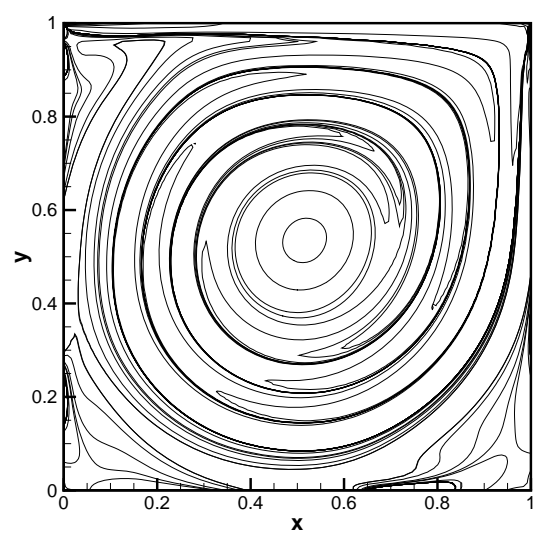

(c)

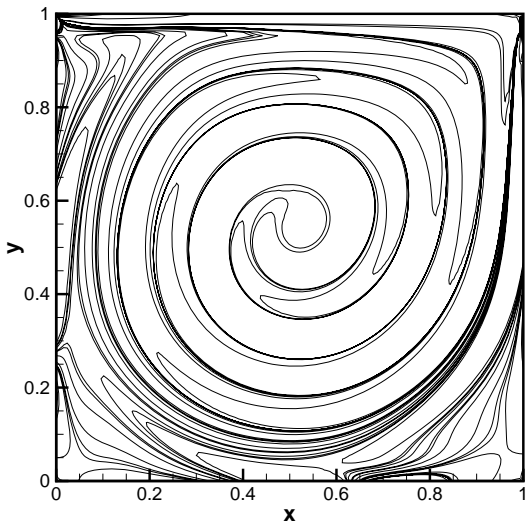

(b)

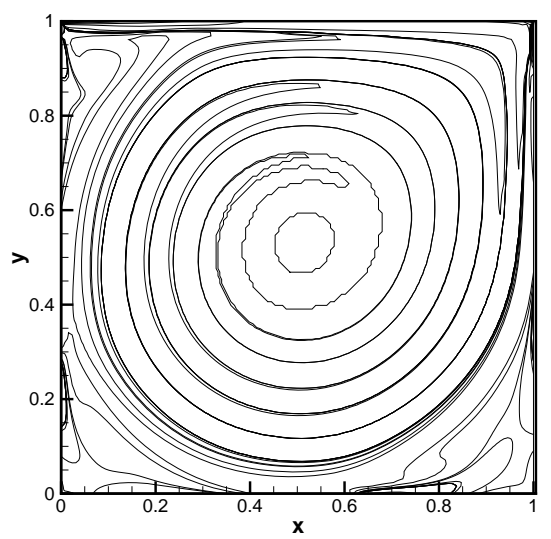

(d)

Figure 5: The steady-state vorticity contours: (a) $R e=1000$; (b) $R e=3200$; (c) $R e=5000$; and (d) $R e=7500$.

Table 7 lists comparison of the velocity results of the $4^{\text {th }}$ order explicit scheme with the present scheme for lid-driven cavity flow with $1000 \leq R e \leq 7500$ and the percentage difference with respect to the benchmark solution [24] with grid of $65 \times 65$. It is clear that the computed solutions from the present methods are much closer to the benchmark solution [24] than those from the $4^{\text {th }}$ order explicit scheme with the same Reynolds numbers. The results in Table 7 also exhibit the superiority of the present method in accuracy over the $4^{\text {th }}$ order explicit scheme with $1000 \leq R e \leq 7500$.

\section{Conclusions}

In this work, we have developed a consistent fourth-order boundary scheme for the two-dimensional incompressible vorticity-stream function form of Navier-Stokes equations. For solving the convection diffusion equation,diffusion and convection term for interior 
Table 5: The minimum values of the velocity component $u$ along $x=0.5$ and the corresponding $y$ coordinate $y_{\min }$, the maximum values of the velocity component $v$ along $y=0.5$ and the corresponding $x$-coordinate $x_{\max }$, and the stream function $\psi$ and the location coordinates $(x, y)$ of the center of primary vortex for different Reynolds numbers $1000 \leq R e \leq 7500$. (Notes: 1 . Solved with $65 \times 65$ grid; 2. Solved with $129 \times 129$ grid).

\begin{tabular}{|c|c|c|c|c|c|c|c|c|}
\hline$R e$ & Reference & $u_{\min }$ & $y_{\min }$ & $v_{\max }$ & $x_{\max }$ & $\psi$ & $x$ & $y$ \\
\hline \multirow{4}{*}{1000} & present $^{1}$ & -0.38242 & 0.1718 & 0.36911 & 0.1562 & -0.117 & 0.5312 & 0.5625 \\
\hline & present $^{2}$ & -0.38690 & 0.1718 & 0.37445 & 0.1562 & -0.118 & 0.5312 & 0.5625 \\
\hline & $\begin{array}{l}\text { Ghia } \\
\text { et al. [24] }\end{array}$ & -0.38289 & 0.1719 & 0.37095 & 0.1563 & -0.117 & 0.5313 & 0.5625 \\
\hline & $\begin{array}{l}\text { Bruneau } \\
\text { et al. [25] }\end{array}$ & -0.37640 & 0.1602 & 0.36650 & 0.1523 & -0.118 & 0.5313 & 0.5639 \\
\hline \multirow{4}{*}{3200} & present $^{1}$ & -0.42068 & 0.1016 & 0.42132 & 0.0937 & -0.120 & 0.5156 & 0.5312 \\
\hline & present $^{2}$ & -0.43235 & 0.0937 & 0.42775 & 0.0937 & -0.120 & 0.5156 & 0.5468 \\
\hline & $\begin{array}{c}\text { Ghia } \\
\text { et al. [24] }\end{array}$ & -0.41933 & 0.1016 & 0.42768 & 0.0938 & -0.120 & 0.5156 & 0.5469 \\
\hline & $\begin{array}{c}\text { Sahin } \\
\text { et al. [29] }\end{array}$ & -0.43540 & 0.0921 & 0.43245 & 0.0972 & -0.121 & 0.5201 & 0.5376 \\
\hline \multirow{4}{*}{5000} & present $^{1}$ & -0.42306 & 0.0703 & 0.42024 & 0.0781 & -0.117 & 0.5156 & 0.5312 \\
\hline & present $^{2}$ & -0.44337 & 0.0781 & 0.44218 & 0.0781 & -0.121 & 0.5156 & 0.5312 \\
\hline & $\begin{array}{c}\text { Ghia } \\
\text { et al. }[24]\end{array}$ & -0.43643 & 0.0703 & 0.43648 & 0.0781 & -0.118 & 0.5117 & 0.5352 \\
\hline & $\begin{array}{l}\text { Bruneau } \\
\text { et al. [25] }\end{array}$ & -0.43590 & 0.0664 & 0.42590 & 0.0762 & -0.114 & 0.5156 & 0.5313 \\
\hline \multirow{4}{*}{7500} & present $^{1}$ & -0.41510 & 0.0625 & 0.40427 & 0.0703 & -0.111 & 0.5156 & 0.5312 \\
\hline & present $^{2}$ & -0.45429 & 0.0625 & 0.45583 & 0.0703 & -0.122 & 0.5156 & 0.5312 \\
\hline & $\begin{array}{c}\text { Ghia } \\
\text { et al. [24] }\end{array}$ & -0.43590 & 0.0625 & 0.44030 & 0.0703 & -0.119 & 0.5117 & 0.5311 \\
\hline & $\begin{array}{l}\text { Bruneau } \\
\text { et al. [25] }\end{array}$ & -0.43790 & 0.0508 & 0.41790 & 0.0625 & -0.111 & 0.5156 & 0.5234 \\
\hline
\end{tabular}

points are used with the fourth-order compact scheme. Then, we proved that there are the consistent fourth-order boundary scheme matching with the interior points scheme. The scheme ensures main truncation error of boundary point scheme and that of interior point scheme remain identical and the coefficient matrix of the jointed boundary and interior schemes is diagonally dominant, which makes algebraic equations have good stability and convergent property. Numerical experiments were performed to demonstrate high accuracy and stability of the present method.

Acknowledgments This work was supported by the National Natural science Foundation of China under Grant (No. 11601013, 91530325) and Foundational Research of Civil Aircraft (No. MJ-F-2012-04)

\section{References}

[1] M. Li, T. TANG AND B. FornBerg, A compact fourth-order finite diffference scheme for the incom- 
Table 6: Comparison of the results secondary vortex at different Reynolds numbers.

\begin{tabular}{|c|c|c|c|c|c|c|c|}
\hline \multirow[b]{2}{*}{$\operatorname{Re}$} & \multirow[b]{2}{*}{ Reference } & \multirow{2}{*}{$\begin{array}{c}\text { Bottom } \\
\psi\end{array}$} & \multicolumn{2}{|c|}{ left } & \multirow{2}{*}{$\frac{\text { Bottom }}{\psi}$} & \multicolumn{2}{|c|}{ right } \\
\hline & & & $x$ & $y$ & & $x$ & $y$ \\
\hline \multirow{5}{*}{1000} & present $^{1}$ & $2.15(-4)$ & 0.0859 & 0.0781 & $1.67(-3)$ & 0.8593 & 0.1093 \\
\hline & present $^{2}$ & $2.31(-4)$ & 0.0859 & 0.0781 & $1.72(-3)$ & 0.8593 & 0.1093 \\
\hline & Ghia et al. [24] & $2.31(-4)$ & 0.0859 & 0.0781 & $1.75(-3)$ & 0.8594 & 0.1094 \\
\hline & Bruneau et al. [25] & $3.25(-4)$ & 0.0859 & 0.0820 & $1.91(-3)$ & 0.8711 & 0.1094 \\
\hline & Gupta and Kalita [27] & $2.02(-3)$ & 0.0875 & 0.0750 & $1.70(-3)$ & 0.8625 & 0.1125 \\
\hline \multirow{4}{*}{3200} & present $^{1}$ & $1.07(-3)$ & 0.0859 & 0.1094 & $2.34(-3)$ & 0.8125 & 0.0859 \\
\hline & present $^{2}$ & $1.05(-3)$ & 0.0859 & 0.1094 & $2.96(-3)$ & 0.8023 & 0.0859 \\
\hline & Ghia et al. [24] & $1.04(-3)$ & 0.0859 & 0.1094 & $2.71(-3)$ & 0.8125 & 0.0859 \\
\hline & Gupta and Kalita [27] & $1.03(-3)$ & 0.0813 & 0.1188 & $2.86(-3)$ & 0.8125 & 0.0875 \\
\hline \multirow{5}{*}{5000} & present $^{1}$ & $1.31(-3)$ & 0.0703 & 0.1406 & $2.48(-3)$ & 0.8125 & 0.0703 \\
\hline & present $^{2}$ & $1.35(-3)$ & 0.0703 & 0.1406 & $3.01(-3)$ & 0.8047 & 0.0703 \\
\hline & Ghia et al. [24] & $1.36(-3)$ & 0.0703 & 0.1367 & $3.08(-3)$ & 0.8056 & 0.0742 \\
\hline & Bruneau et al. [25] & $2.22(-3)$ & 0.0664 & 0.1484 & $4.65(-3)$ & 0.8301 & 0.0703 \\
\hline & Gupta and Kalita [27] & $1.32(-3)$ & 0.0750 & 0.1313 & $2.96(-3)$ & 0.8000 & 0.0750 \\
\hline \multirow{5}{*}{7500} & present $^{1}$ & $1.42(-3)$ & 0.0625 & 0.1562 & $2.12(-3)$ & 0.7812 & 0.0625 \\
\hline & present $^{2}$ & $1.53(-3)$ & 0.0625 & 0.1562 & $3.10(-3)$ & 0.7812 & 0.0625 \\
\hline & Ghia et al. [24] & $1.47(-3)$ & 0.0645 & 0.1504 & $3.28(-3)$ & 0.7813 & 0.0625 \\
\hline & Bruneau et al. [25] & $4.76(-3)$ & 0.0703 & 0.1289 & $8.32(-3)$ & 0.8828 & 0.0820 \\
\hline & Gupta and Kalita [27] & $1.60(-3)$ & 0.0688 & 0.1500 & $3.05(-3)$ & 0.7813 & 0.0625 \\
\hline
\end{tabular}

Table 7: Comparison of the velocity results of the $4^{\text {th }}$ order explicit scheme with the present scheme for lid-driven cavity flow at $1000 \leq R e \leq 7500$ and the percentage difference with respect to the benchmark solution [24] with grid of $65 \times 65$.

\begin{tabular}{||cccccc||}
\hline Re & method & $u_{\min }$ & difference(\%) & $v_{\max }$ & difference(\%) \\
\hline \multirow{2}{*}{1000} & $4^{\text {th }}$ order explicit & -0.38170 & -0.3107 & 0.36840 & -0.6874 \\
& present & -0.38242 & -0.1227 & 0.36911 & -0.4902 \\
\multirow{3}{*}{3200} & $4^{t h}$ order explicit & -0.42582 & -1.5429 & 0.41610 & -2.7071 \\
& present & -0.42068 & -0.3219 & 0.42132 & -1.4870 \\
\multirow{2}{*}{5000} & $4^{t h}$ order explicit & -0.41546 & -4.8048 & 0.41593 & -4.7081 \\
& present & -0.42306 & -3.0634 & 0.42024 & -3.7206 \\
7500 & $4^{t h}$ order explicit & -0.40612 & -6.8364 & 0.40350 & -8.8373 \\
& present & -0.41510 & -4.7717 & 0.40427 & -8.1810 \\
\hline \multirow{2}{*}{} & & & & &
\end{tabular}

pressible Navier-Stokes equations, Int. J. Numer. Meth. Fl., 20 (1995), pp. 1137-1151.

[2] M. Li And T. TANG, A compact fourth-order finite difference scheme for unsteady viscous incompressible flows, J. Sci. Comput., 16 (2001), pp. 29-45.

[3] Y. Ma, D. Fu, T. Kobarash AND N. TANiguchi, Numerical solution of the incompressible NavvierStokes equations with an upwind compact difference scheme, Int. J. Numer. Meth. Fl., 30 (1999), pp. 509-521. 
[4] W. F. Spotz, G. F. CAReY, High-order compact scheme for the steady stream-functionvorticity equations, Int. J. Numer. Meth. Eng., 38 (1995), pp. 3497-3512.

[5] Y. Sanyasiraju and V. Manjula Higher order semi compact scheme to solve transie incompressible Navier-Stokes equations, Comput. Mech., 35 (2005), pp. 441-448.

[6] Z. F. TIAn AND Y.B. GE, A fourth-order compact finite difference scheme for the steady streamfunction-vorticity formulation of the Navier-Stokes/Boussinesq equations, Int. J. Numer. Meth. Fl., 41 (2003), pp. 495-518.

[7] S. P. Ferreira, J. Pereira, Fourth- and tenth-order compact finite difference solutions of perturbed circular vortex flows, Int. J. Numer. Meth. Fl., 49 (2005), pp. 603-618.

[8] R. S. HiRsh, Highter order accurate difference solutions of fluid mechanics problems by a compact differencing technique, J. Comput. Phys., 19 (1975), pp. 90-109.

[9] S. K. LeLE, Compact finite difference schemes with spectral-like resolution, J. Comput. Phys., 103(1) (1992), pp. 16-42.

[10] M. Yanwen and F. Dexun, Numerical solution of the incompressible Navier-Stokes equations with an upwind compact difference scheme, Int. J. Numer. Meth. Fl., 30 (1999), pp. 509-521.

[11] P. C. Chu AND C. FAN, A three-point combined compact difference scheme, J. Comput. Phys., 140 (1998), pp. 370-399.

[12] P. C. CHU AND C. FAN, A three-piont sixth-point sixth-order nonuniform combined compact difference scheme, J. Comput. Phys., 148 (1999), pp. 663-674.

[13] Z. TIAN, Y. A. LI, Numerical solution of the incompressible Navier-Stokes equations with a threepoint fourth-order upwind compact difference schemes, Fourth International Conference Nonlinear Mechanic, 2002, pp. 942-948.

[14] K. K. Q. Zhang And B. Shotorban, A compact finite difference method on staggered grid for Navier-Stokes flows, Int. J. Numer. Meth. Fl., 2006, pp. 1-24.

[15] X. Liu, S. H. Zhang, H. X. Zhang And C. W. Shu, A new class of central compact schemes with spectral-like resolution I Linear schemes, J. Comput. Phys., 248 (2013), pp. 235-256.

[16] B. GUSTAFSSON, The convergence rate for difference approximations to mixed initial boundary value problems, Math. Comput., 29 (1975), pp. 396-406.

[17] M. CARPENTER, The stability of numerical boundary treatments for compact high-order finitedifference schemes, J. Comput. Phys., 108 (1993), pp. 272-295.

[18] W. E. Weinan and J. Liu, Essentially Compact Schemes for Unsteady Viscous Incompressible Flows, J. Comput. Phys., 126 (1996), pp. 122-138.

[19] W. E. Weinan And J. Liu, Vorticity Boundary Condition and Related Issues for Finite Difference Schemes, J. Comput. Phys., 124 (1996), pp. 368-382.

[20] C. W. SHU AND S. OsHeR, Efficient implementation of essentially non-oscillatory shock capturing Schemes, J. Comput. Phys., 2(77) (1988), pp. 439-471.

[21] Z. TIAN AND S. DAI, High-order compact exponential finite difference methods for convectiondiffusion type problems, J. Comput. Phys., 220 (2007), pp. 952-974.

[22] C. W. RichaRDS AND C. W. CRANE, The accuracy of finite difference schemes for the numerical solution of the Navier-Stokes equations, J. Comput. Phys., 220 (2007), pp. 952-974.

[23] C. W. Richards AND C. W. CRANE, The accuracy of finite difference schemes for the numerical solution of the Navier-Stokes equations, J. Comput. Phys., 220 (2007), pp. 952-974.

[24] U. GHIA, K. N. GHIA AND C. T. SHIN, High-Re solutions for inconpressible flow using the NavierStokes rquations and a multigrid method, J. Comput. Phys., 48 (1982), pp. 387-411.

[25] C. H. BRunEAu AND C. Jouron, An effcient scheme for solving steady incompressible NavierStokes equations, J. Comput. Phys., 89 (1990), pp. 389-413.

[26] S. Hou, Q. Zou, S. Chen, G. Doonlen AND A. C. Cogely, Simulation of cavity flow by the Lattice Boltzmanne method, J. Comput. Phys., 118 (1995), pp. 329-347. 
[27] M. M. Gupta And J. C. Kalita, A new paradigm for solving Navier-Stokes equations: streamfunction-velocity formulation, J. Comput. Phys., 207 (2005), pp. 52-68.

[28] V. Stephane, A 2D compact fourth-order projection decomposition method, J. Comput. Phys., 206 (2005), pp. 252-276.

[29] M. SAHIN AND R. G. OWENS, A novel fully impicit finit volume method applied to the lid-driven cavity problem-Part I:High Reyonds number flow calculations, Int. J. Numer. Meth. Fl., 42 (2003), pp. 57-77. 